\title{
Politiciens de la santé ...
}

Rainer M. Kaelin

Correspondance:

Dr Rainer M. Kaelin Place de l'Hôtel-de-Ville 2 CH-1110 Morges

rmkaelin@sunrise.ch
Le maire de New York, Michael Bloomberg, a reçu, le 5 octobre 2008, à Berlin, le prix de la European Lung Foundation [1] pour ses efforts extraordinaires au service de la santé humaine.

La politique de la santé est le résultat de multiples forces en action. L'argument santé est d'importance relative dans la tactique déployée par les différents groupes d'intérêts dont le spectre couvre l'industrie pharmaceutique, les professions de la santé, le secteur économique, celui de l'alimentation, en passant par les assureurs, les caisses-maladie, les fabricants d'instruments diagnostiques et thérapeutiques, les hôpitaux, les fonctionnaires de la santé publique, jusqu'aux représentants du peuple dans les parlements, qui, tous, la bouche en cœur, affirment avec conviction vouloir défendre le bien commun.

Le politicien sans parti, Michael Bloomberg, dont la fortune privée est estimée à 20 milliards de dollars américains, n'est pas - indubitablement - un taliban de la santé; il ne cache pas son ambition de décrocher un troisième mandat à la tête de la métropole [2]. Il n'en était cependant pas question, lorsqu'il a reçu son prix au congrès annuel [3] de l'European Respiratory Society. Suivent des extraits de son allocution:

«J'aimerais vous parler de ce pourquoi je m'engage autant comme fonctionnaire que comme philanthrope privé et de ce que vous devez faire encore plus vigoureusement et plus efficacement que ce que vous faites aujourd'hui en tant que chercheurs et médecins: enrayer la première cause de mort évitable du globe, une cause plus meurtrière que la tuberculose, le sida et le paludisme pris ensemble, qui tue 5 millions de personnes par année dans le monde: le tabac. [...] C'est une calamité de premier ordre pour la santé publique; mais rien n'est inéluctable. Elle peut être évitée si les gouvernements du monde agissent pour la défier. La bonne nouvelle est que nous savons ce que nous avons à faire. La convention-cadre pour le contrôle du tabagisme [4], le premier traité international de santé publique, développé par la lauréate de l'European Lung Foundation de 2003, le Dr Gro Harlem Brundtland [5], a été ratifié par 150 nations [...]

Il y a trois mois, notre Fondation a versé à nouveau 250 millions de dollars américains, pour les quatre prochaines années, à l'Initiative pour réduire la consommation du tabac [6]). Elle est soutenue aussi par la Fondation de Bill et Melinda Gates et l'OMS. [...] Je suis persuadé que la meilleure manière de mener le mouvement n'est pas seulement l'exhortation mais aussi l'exemple. L'urgence d'agir va au-delà des frontières, et la responsabilité de conduire l'action pèse plus lourdement sur les épaules de ceux qui disposent des moyens de l'assumer. [...]
Il y a six ans, nous avons libéré New York de la fumée. Nous étions face à une opposition énorme. On nous prédisait, que les touristes, spécialement les Européens, allaient éviter notre ville. Aujourd'hui, les New Yorkais considèrent notre législation «libre de fumée» comme allant de soi. Et le tourisme international, dont celui de l'Europe, atteint un niveau record. Lorsque nous avons introduit notre loi, seulement un Etat sur 50 était libre de fumée. Maintenant la plupart des Américains vivent dans des juridictions où la fumée est bannie. Et, autour du globe, des villes comme Paris, des pays comme l'Italie, la Norvège, l'Angleterre, l'Irlande et la Turquie ont suivi. Je suis heureux de vous dire que l'Inde, la plus grande démocratie du monde, a joint cette liste il y a peu. [...]

Ici, en Allemagne, saisissez l'opportunité qui se présente, suite à la décision de la Cour constitutionnelle [7] qui confirme le droit d'interdire la fumée dans les bars et restaurants, pour promouvoir une loi fédérale bannissant la fumée dans tous les espaces publics fermés du pays. [...] Redoublez vos efforts! Soyez conscients des paroles du père de la médecine, Hippocrate [...]. Le devoir de protéger et promouvoir la santé est bien supérieur à celui de la réparer une fois qu'elle est atteinte. En d'autres termes, vous, médecins, vous avez un rôle important à jouer en tant qu'acteurs d'une santé publique efficace [...].»

Jusqu'à présent, aucun des acteurs de la santé publique suisse ne s'est positionné comme l'a fait le maire Bloomberg. La discrétion n'exclut cependant pas l'action lourde de conséquences, comme le démontre l'exemple de Christoffel Brändli [8], ancien président de santésuisse et du Conseil des Etats. En décembre 2007, il avait, par vote présidentiel, validé l'article constitutionnel sur les caisses-maladie dans un Conseil des Etats partagé (article, qui a été rejeté par le peuple en juin 2008, suite au referendum lancé par la FMH). Sa position d'alors était compréhensible, puisque les assureurs auraient obtenu par cet amendement un avantage décisif dans le système de santé suisse [9]. Presque une année plus tard, le même politicien de la santé, à nouveau par vote présidentiel, a fait que la chambre haute accepte l'actuelle loi fédérale, vidée de sa substance par les exceptions concernant la protection contre la fumée passive (voir encadré). Dans ce dernier cas, il n'est pas très clair à qui a profité la décision courageuse! Certainement pas à la santé des clients et employés des établissements publics ni aux caisses- maladie ... Néanmoins, il est tout aussi improbable que son hochement de tête fut le fruit du hasard [10]. 
- Dr. med. Jürg Barben, Chefarzt pädiatrische Pneumologie, Ostschweizer Kinderspital St. Gallen

- Prof. Dr. med. Martin Brutsche, Chefarzt Pneumologie, Kantonsspital St. Gallen

- Dr. med. Otto Brändli, Pneumologie und Innere Medizin FMH, Präsident Lungenliga Zürich

- Prof. Dr. med. Thomas Cerny, Chefarzt Onkologie, Kantonsspital St. Gallen, Präsident Krebsliga Schweiz

- Prof. Jacques Cornuz, Policlinique médicale universitaire, président CIPRET, Lausanne

- Dr. med. Martin Frey, Chefarzt Klinik Barmelweid

- Dr Rainer M. Kaelin, médecine interne et pneumologie FMH, vice-président Ligue pulmonaire vaudoise, Morges

- Dr. med. Werner Karrer, Präsident Schweizerische Gesellschaft für Pneumologie, Vizepräsident Lungenliga Schweiz, Chefarzt Luzerner Höhenklinik Montana

\section{Loi fédérale pour la protection de la population contre la fumée passive, du 3. octobre 2008}

[Traduction R. M. Kaelin; il n'existe pas de version française officielle actuellement.]

\section{Art. 1 Domaine d'application}

$\S 1$. Cette loi concerne la protection contre la fumée passive dans les espaces publics fermés, accessibles au public ou servant de lieu de travail à plusieurs personnes. § 2. En tant qu'espaces publics fermés sont considérés en particulier: a. Bâtiments de l'administration publique; b. Hôpitaux et autres institutions de soins; c. Garderies d' enfants, établissements médico-sociaux pour personnes âgées et institutions assimilables; e. Etablissements pour l'enseignement et la formation; f. Musées, théâtres, cinémas; g. Installations sportives; $\mathbf{h}$. Etablissements dédiés à l'hôtellerie et à la restauration, [...] indépendamment du règlement cantonal des permis d'exploitations. i. Bâtiments et véhicules de transport public; j. Commerces et centres commerciaux. §3. Les locaux à usage privé ne sont pas soumis à la présente loi.

Art. 2 Interdiction de fumer

$\S 1$. Il est interdit de fumer dans les espaces définis en Art. $1, \S 1$ et 2 . Le gérant ou la personne responsable du règlement de maison peut permettre qu'on fume dans des espaces spécialement aménagés, dans lesquels aucun employé ne travaille, pour autant que ces espaces («fumoirs») soient séparés des autres espaces, clairement signalés comme tels et munis d'une ventilation adéquate. Exceptionnellement, et seulement avec leur accord explicite, des employés peuvent travailler dans les
- Prof. Laurent Nicod, médecin-chef, Service de pneumologie, CHUV, Lausanne, Chairman European Lung foundation 2004-2007

- Dr. med. Maurus Pfister, Pneumologie FMH, Rorschach

- Dott. Franco Quadri, capo pneumologia, Ospedale Bellinzona e Valli, Bellinzona

- Prof. Thierry Rochat, médecin-chef du Service de pneumologie, Hôpital cantonal universitaire de Genève, Principal investigator étude SAPALDIA

- Dr. med. M. Schuurmans, Pneumologie FMH, Claraspital, Basel

- Dr Olivier Staneczek, Pneumologie FMH, Montreux

- Prof. Jean-Marie Tschopp, médecin-directeur Centre valaisan de Pneumologie, chef du Département de médecine interne du CHCVs

- Dr Jean-Pierre Zellweger, privat-docent, pneumologie FMH, Fribourg

fumoirs dans les secteurs de l'hôtellerie et de la restauration. L'accord des employés doit être partie intégrante du contrat de travail. § 3 . Le Conseil fédéral édicte les ordonnances concernant la conception et les exigences de la ventilation. II règle également les conditions applicables dans les institutions pour exécutions de peines et là, où des personnes sont dans l'obligation de séjourner de manière permanente ou pour une longue durée.

\section{Art. 3 Etablissements fumeurs}

Sur demande, un permis est accordé pour des établissements fumeurs qui remplissent les conditions suivantes: a. Ils disposent d'une surface accessible au public de maximum $80 \mathrm{~m}^{2}$; b. Ils disposent d'une ventilation adéquate et sont clairement reconnaissables comme établissements fumeurs; c. Ils emploient exclusivement des personnes ayant accepté, dans le contrat de travail, l'activité dans un établissement fumeur.

Art. 4 Dispositions cantonales

Les Cantons peuvent édicter des mesures plus contraignantes pour la protection de la santé.

Art. 5 Dispositions pénales

Art. 6 Dispositions d'exécution

Art. 7 Referendum et mise en vigueur

Signé pour le Conseil national: André Bugnon; pour le Conseil des Etats: Christoffel Brändli.

[La loi a été acceptée au Conseil national par 89 oui contre 88 non, et au Conseil des Etats par la décision du président.] 


\section{Notes et références}

1 L'European Lung Foundation (ELF) est une fondation de la société des pneumologues européens (European Respiratory Society ERS), dont le congrès annuel 2008, à Berlin, a réuni plus de 10000 participants. Elle remet chaque année un prix à des personnalités pour des actions extraordinaires au service de la santé publique dans le domaine de la respiration. Parmi les lauréats précédents, il faut mentionner $\mathrm{M}^{\mathrm{me}}$ le Docteur Gro Harlem Brundtland [5] et Sir Richard Peto pour ses travaux de pionnier sur la mortalité du cancer en relation avec le tabagisme et la prévention du cancer. Le maire Bloomberg reçoit le prix 2008, car, le premier, il a démontré que des villes peuvent devenir sans fumée en interdisant de fumer sur les lieux de travail. Il se fait le promoteur de cette approche pour d'autres villes dans le monde et a versé 375 millions de dollars américains dans la lutte contre le tabagisme dans les pays en développement et dans plusieurs pays de l'Europe de l'Est.

2 Suter M. Michael Bloomberg will dritte Amtszeit. New Yorks Bürgermeister sieht sich als Retter der Stadt. SonntagsZeitung, 12. Oktober 2008. Il est à noter, qu'à notre connaissance, aucun journal suisse parlant du maire de New York n'a mentionné son activité pour une ville libre de fumée, son «Initiative pour réduire la consommation du tabac» et son prix de l'ELF.

3 Le texte intégral de l'allocution du Maire Bloomberg se trouve dans le Périodique de la Fondation ELF, «Breath» (2008;5[2]:117-19). Il peut être téléchargé du site www.european-lung-foundation.org.

4 La Framework Convention Tobacco Control (FCTC) (en français: Convention cadre pour le contrôle du tabac) est une convention internationale ratifiée par 150 pays. La Suisse l'a signée, mais pas ratifiée. Elle est importante, parce qu'elle oblige les partenaires (les pays) d'appliquer les mesures essentielles dans la lutte contre le tabagisme, tout en réglant la collaboration internationale et l'échange d'informations dans ce domaine. Sa ratification était prévue durant la législature actuelle des parlements fédéraux suisses, mais la loi du 3 octobre 2008 (voir encadré) en est un obstacle. Voir aussi note 9, Kaelin RM. La convention cadre du contrôle du tabac a bientôt 5 ans. Bull Méd Suisses. 2008;89(22):968-71, et www.who.int/tobacco/framework.

5 Dr Gro Harlem Brundtland fut Premier Ministre norvégien en 1981, 1986-1989 et 1990-1996 puis Directrice de l'OMS dès 1998. Pendant cette période, le Prof. Zeltner, Directeur de l’Office fédéral de la santé publique, a été mandaté comme expert pour investiguer les efforts de l'industrie du tabac visant à miner les mesures de l'OMS dans la lutte pour le contrôle du tabagisme dans le monde.

6 L'Initiative pour réduire l'utilisation du tabac a été lancée par Michael Bloomberg en 2005 et l'a dotée d'un fonds de 125 millions de dollars américains. Elle collabore avec l'OMS, la Bloomberg School of Public Health de l'université Johns Hopkins et d'autres partenaires, dans le but d'investiguer l'étendue de l'épidémie globale du tabagisme et de développer des stratégies de santé publique pour la combattre.

7 Le verdict de la Cour constitutionnelle allemande du 30 juillet 2008 invalide les lois contre la fumée passive de Bade-Wurtemberg et de Berlin, car elles violent le principe d'égalité de traitement devant la loi des restaurateurs. Mais, les considérants de cette décision confirment sans équivoque, que l'inégalité de traitement prend son origine dans les exceptions à la règle du principe selon lequel les êtres humains ont le droit d'être protégés de la fumée passive. Une application stricte de la règle de protection n'est pas en contradiction avec la constitution allemande et elle est en accord avec la convention cadre pour le contrôle du tabagisme de l'OMS ratifiée par l'Allemagne.

8 Christoffel Brändli est politicien UDC et représente le Canton des Grisons au Conseil des Etats, dont il fut président de décembre 2007 à décembre 2008. Jusqu'en 2008, il était également président de santésuisse. Selon ses propres déclarations (www. parlament.ch; Janvier 2008), il a les mandats suivants: Beton und Kies, Unterrealta: président du Conseil d'administration; HTM Immobilien, Passugg: Conseil d'administration; Rätia Energie SA. Brusio: Conseil d'administration; Ecole Suisse du tourisme et hôtellerie SA, Passugg: Conseil d'administration; STAG SA, Maienfeld: Conseil d'administration; Walo, entreprise de construction SA, Coire: présiden du Conseil d'administration; Bureau de conciliation des assurance sociales, Lucerne: membre.

9 Concernant la séparation des pouvoirs dans la politique de la santé, voir de Haller J. L'esprit des lois ou la séparation des pouvoirs ... Bull Méd Suisses. 2009;90(8):283, et Kuhn HP. Il ne faut pas que les contrôleurs se contrôlent eux-mêmes. Bull Méd Suisses. 2009;90(8):285.

10 Arsever S. Ecrire l'Histoire. Commentaire. Le Temps du 3.10.2008. 